\title{
Estimating Complex Refractive Index Using Ellipsometry
}

\author{
Gul e Saman and Edwin R. Hancock ${ }^{\star}$ \\ Department of Computer Science, University of York, YO10 5GH, UK
}

\begin{abstract}
The paper is about estimating complex refractive index images of surfaces. Complex refractive index allows both the transmission and absorption properties of a surface to be characterised. Although widely used in applied optics, there has been little work on its use in image processing and surface inspection. In this paper we describe how to compute the complex refractive index of a material using simple digital image measurements. Specifically, a novel technique for estimating complex refractive index using ellipsometry is reported. The method uses a simple experimental setup that relies on the use of raw intensity data. This represents an advantage over the methods used in applied optics. Rather than relying on the calibrated measurement of absorption, instead our method relies on measuring the relative change in reflection amplitude which is a dimensionless quantity.
\end{abstract}

Keywords: Complex Refractive Index, Absorption, Extinction Co-efficient, Naturally occurring surfaces, Manufactured surfaces, Photometric stereo, Fresnel Theory.

\section{Introduction}

When incident light is scattered from a surface, then there is always partial absorption. Classical refractive index does not fully capture the situation where scattering involves both absorption and transmission, since it measures only the ratio of the speed of light in material to that in a vacuum. Complex refractive index, on the other hand, can be used to describe both the transmission and absorption properties of naturally occurring surfaces. Such a characterisation is essential for diagnostic purposes in naturally occurring biological tissues.

Complex refractive index is a composite quantity comprised of a real part that gives the refractive index and the imaginary part which gives the extinction co-efficient for the material, and is given as $m=n+i \alpha$. where $n$ is the refractive index of the scatterer and $\alpha$ is the extinction co-efficient which is used to determine absorption. The quantity can be directly measured using ellipsometry for planar samples. For naturally occurring materials, chemical suspensions must be prepared, and then ellipsometry applied.

The refractive index of a naturally occurring surface is a basic quantity that determines the interaction of light and its propagation in the medium. As most types of naturally occurring surface are inhomogeneous and optically turbid, the methods that

\footnotetext{
* Edwin Hancock is supported by the EU FET project SIMBAD and by a Royal Society Wolfson Research Merit Award.
} 
have been employed thus far to determine the refractive index of materials cannot be applied to such surfaces. Methods involving the use of refractometers for determining the refractive index require large optical contact areas between the sample and the instrument. The refractive index of many kinds of naturally occurring tissues are hence unmeasurable using refractometers. Refractive index is key to understanding how light propagates in a material and knowledge about the refractive index of a naturally occurring surface is important for optical diagnosis and treatment. The refractive index of a biological tissue can be measured by cladding an optic fiber with a tissue and measuring the cone angle of the transmitted light. In this way the refractive indices for different kinds of mammalian tissues have been measured at a wavelength of $632.8 \mathrm{~nm}$ [1]. Moreover, the refractive indices of thin samples of dehydrated human skin have been measured using a laser refractometers based on total internal reflection. To determine the refractive index of fresh hydrated tissues, the values obtained from the dehydrated tissues must be modified based on estimates of the water content [2].

The above methods can only determine the real part of the complex refractive index of a material and this does not incorporate the absorption of light by the material.

Confocal microscopy has also been used in order to measure the refractive index of tissues [3]. Using a layer of immersion fluid of exactly the same depth as the tissue, it takes into account the 3-D point spread function (PSF) of the microscope. Greenleaf et al. [4], have used computerised tomography to compute two images for the refractive index $n$ and acoustic attenuation $\alpha$ representation. The values have been computed using profiles of propagation times and amplitudes of digitized acoustic pulses of tissues viewed at multiple angles from rectilinear transmission scans. It might be considered intrinsically more valuable to have quantitative data than qualitative data, as it gives more information about the variation in the absorption of the material and also in the refractive index.

There are many methods for computing the complex refractive index of a material such as ellipsometry and reflectance versus incidence angle methods [5]. Ding et al. [6], used the automated reflectometer system that was developed to determine the refractive index of a turbid sample by measuring the coherent reflectance $(R)$ vs the angle of incidence $(\theta)$. The Fresnel equations have been used in conjunction with non-linear regression $R(\theta)$ to determine the complex refractive index of human skin. It has been determined for eight wavelengths of both the dermis and the epidermis tissues. The reflectance of $\mathrm{p}$ and $\mathrm{s}$ polarisation is measured at different angles of incidence or polarisation conditions. These are related to the variation of the light intensity. The results are affected by the scattering light, internal reflection and other factors.

The aim in this paper is to develop a more direct surface inspection method for determining complex refractive index using the type of equipment commonly found in a machine vision laboratory. Specifically our set up consists of an ellipsometry prism placed at the centre of an geodesic light dome. Polarised light sources placed at the nodes of the dome, illuminate a sample of material. Images collected using a digital camera and a polaroid analyser, can be used to make measurements of complex refractive index. Moreover, the method provides for the first time a means of producing a complex refractive index image of the surface, which can be used to segment surfaces into regions of different composition or to inspect for surface lesions or flaws. 

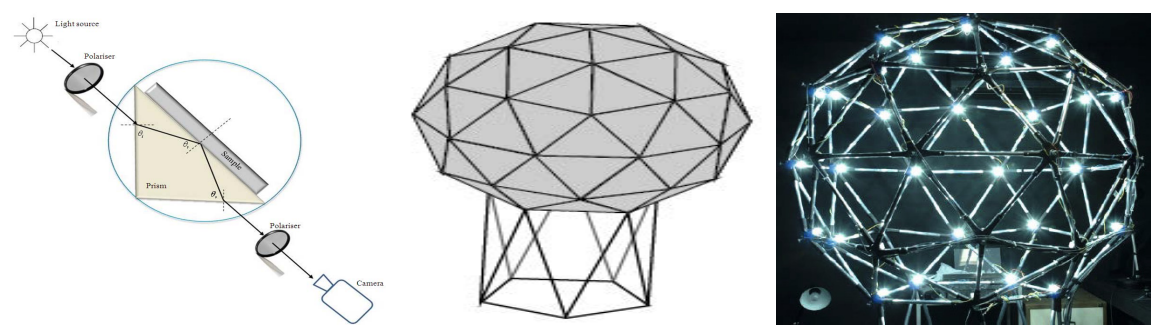

Fig. 1. The experimental setup for complex refractive index estimation using ellipsometry (left), light dome (center and right)

\section{Polarised Light}

Unpolarised light incident on a surface acquires partial polarisation due to the orientation of the dipoles in the scatterer. Polarisation information can be used for analysing small changes in a surface. Partially linearly polarised light has information that can be used for various purposes, for example leaves of different plants reflect light with a varying degree of polarisation [8]. The use of polarisation information by animals for navigation can be largely divided into two categories 1 . macro-scale-orientation and 2 . micro-scale-orientation. The macro-scale-orientation can be used to extract information about larger objects such as, trees and tree tops while, for the micro-scale-orientation information about smaller objects such as individual leaves [7].

\section{Ellipsometry}

Ellipsometry is the measurement of the effect of reflection on the state of polarisation of light. The method involves the measurement and interpretation of the change in the state of polarisation, when polarised light is reflected from a scattering surface. It is a method that is used in industry to measure the optical constants of materials and also for measuring the thickness and the optical constants for films placed on materials under study. It is important to characterise light waves incident on scattering surfaces. It is also of significance to understand how the light wave interacts with a reflecting surface and how it changes on contact with it and how can the change be related to the optical properties of a material.

A beam of incident light can be separated into electric field components that are perpendicular $\left(E_{\perp}\right)$ and parallel $\left(E_{\|}\right)$to the plane of incidence. Similarly the perpendicular and parallel components of the reflected electric field are given by $R_{\perp}$ and $R_{\|}$, respectively.

The amplitude reflection co-efficient can be defined in terms of amplitude and phase change given as

$$
r_{\|}=\frac{R_{\|}}{E_{\|}} \exp \left[i\left(\theta_{\| r}-\theta_{\| i}\right)\right]
$$


where $\theta_{\| i}$ and $\theta_{\perp i}$ are the phase angles of the incident light, and $\theta_{\| r}$ and $\theta_{\perp r}$ the phase angles of reflected light. The phase differences $\theta_{\| r}-\theta_{\| i}$ and $\theta_{\perp r}-\theta_{\perp i}$ are not directly measurable. However, the ratio of

where

$$
\begin{gathered}
\frac{r_{\|}}{r_{\perp}}=\frac{\frac{R_{\perp}}{R_{\|}}}{\frac{E_{\|}}{E_{\perp}}} \exp (i \triangle)=\tan \phi \exp (i \triangle) \\
\phi=\arctan \left[\left(\frac{R_{\|}}{R_{\perp}}\right)\left(\frac{E_{\|}}{E_{\perp}}\right)\right]
\end{gathered}
$$

Ellipsometry involves the measurement of $\tan \phi$ and $\triangle$ which are the relative change in amplitude and the phase difference on reflection. Here we deal with materials that absorb light, hence the use of the imaginary part of the refractive index.

\subsection{Data Acquisition}

We collect a succession of images of the different samples using a geodesic light dome constructed along the lines suggested in [9]. A schematic of the dome is shown in the centre of Figure (1) and a photograph of the completely lit dome is also shown in the figure. It consists of a geodesic grid of diameter 1.58 meter with 41 ultra bright white LEDs attached to the vertices of twice sub-divided icosahedron. A rotating polarising filter is placed in front of each LED. The geodesic dome has been used to accurately determine light source positions. The ellipsometry apparatus (essentially a prism) shown at the left of Figure (1) is placed at the centre of the dome. The prism is then imaged through a polaroid analyser using a digital camera, placed at a known viewing angle. Images are collected with the analyser at various angles. The LED polarisers are oriented and images collected as described below.

Various materials have been studied including chemical compound suspensions (industrially produced) and naturally occurring biological tissues. The chemical compounds that were used for creating suspensions for the experimentation are: Zinc Oxide, Graphite and Cobaltus Sulphate (coarse heterogeneous particle size and finely powdered to nearly homogeneous particle size). Painted samples using acrylic paints of different colours mixed with various materials forming new compounds. For the naturally occurring biological tissues, slices of potato, pepper, apple and orange (peel) have been used for estimating the complex refractive index. Slices have been taken due to the small surface area of the base of the prism (as the dimensions have to be comparable) and the requirement of the method for the use of planar samples, which is possible in the case of the chemical compounds, but a challenge in the case of the biological tissues. This is so because the sample has to be in complete contact with the surface of the prism.

\subsection{Complex Refractive Index Using Ellipsometry}

Here the complex refractive index is estimated by using a modification of the method proposed in [10]. This combines reflection ellipsometry with the principle of total reflection. Conventionally, a goniometric table has been used for the setup and recording 
of the observations with a He-Ne laser as a light source. However, in this paper, we have used the geodesic light dome where the LED acts as a light source and the ellipsometry setup (a prism) is placed at the approximate center of the dome. The orientation of the polariser placed in front of the incident light is set to $45^{\circ}$, in order to equally divide the power into the perpendicular $\perp$ and parallel $\|$ polarisation components before it reaches the right angled glass prism (N-BK7) of refractive index 1.517. A camera (Nikon D200 digital SLR) has been used to measure the transmitted intensity $I_{t}$. A polaroid filter is placed in front of the camera, the analyser angle is rotated manually by increments of $10^{\circ}$ or $5^{\circ}$ (in some cases) to give 19 and 37 images per sample, respectively. The orientation of the second polariser is set such that the angle of separation between the two polarisers is $0^{\circ}$. The degree of polarisation state can be computed by using Saman and Hancock's method of moments given in [11], while the angle of incidence is set to $34.12^{\circ}$.

The composite quantity for the complex refractive index ' $\mathrm{m}$ ' is defined as $m=n+i \alpha$ where $\alpha$ is obtained from the extinction co-efficient $k=\frac{4 \pi \alpha}{\lambda}$. The complex refractive index can also be expressed as: $m=n-i \alpha$, and when $\alpha>0$ light is absorbed by the scatterer while in case of $\alpha=0$ light travels without any loss, for the case of $\alpha<0$ the incident light is considered to be amplified. The convention followed for the expressing the complex refractive index during the course of this paper is: $m=n+i \alpha$.

Using the angles of each of the faces of the prism of AB, AC and BC with Snell's Law, the refractive index is given by

$$
n=\sqrt{\left(\left(\frac{q+1}{q-1}\right) n_{0} \frac{\sin ^{2} \theta_{r}}{\cos \theta_{r}}\right)^{2}+n_{0} \sin ^{2} \theta_{r}}
$$

where $q=-\frac{\alpha_{e} e^{-i \Delta}}{\cos ^{2}\left(\theta_{i}-\theta_{i}^{\prime}\right)}, \theta_{i}^{\prime}=\sin ^{-1}\left(\frac{\sin \theta_{i}}{n_{0}}\right), \theta_{r}=\sin ^{-1}\left(\frac{1}{n_{0}} \sin \left(\theta_{i}\right)\right)+45^{\circ}$ and $n_{0}$ is the refractive index of the prism while $\theta_{i}$ is the angle of incidence of the light source. The values for $\alpha_{e}$ and $\triangle$ once computed can be used to measure the complex refractive index of the sample. Ellipsometry is used to determine the values of $\alpha_{e}$ and $\triangle$. The transmitted light intensity $I_{t}$ varies with the angle of the polariser placed in front of the camera, and follows the transmitted radiance sinusoid

$$
I_{t}=\left(I_{0}-I_{1}\right)\left[T_{0}+\sqrt{A_{0}^{2}+B_{0}^{2}} \sin (2 \phi+\beta)\right]
$$

where $T_{0}=\frac{\left|r_{\|}\right|^{2}+\left|r_{\perp}\right|^{2}}{2}, A_{0}=\frac{\left|r_{\|}\right|^{2}-\left|r_{\perp}\right|^{2}}{2}, B_{0}=\left|r_{\|}\right| \cdot\left|r_{\perp}\right| \cos \triangle$, and $\beta=\tan ^{-1} \frac{B_{0}}{A_{0}}=\tan ^{-1} \frac{B_{1}}{A_{1}}$. The extinction co-efficient $\alpha_{e}$ which is given by the dimensionless

$$
\alpha_{e}=\sqrt{\frac{1-\frac{A_{1}}{T_{1}}}{1+\frac{A_{1}}{T_{1}}}}
$$

where $\triangle=\delta_{\|}-\delta_{\perp}=\cos ^{-1}\left(\frac{1-\alpha_{e}^{2}}{2 \alpha_{e}} B_{0} A_{0}\right)=\cos ^{-1}\left(\frac{1-\alpha_{e}^{2}}{2 \alpha_{e}} B_{1} A_{1}\right)$ 
where $r_{\perp}$ and $r_{\|}$are the amplitude transmission coefficients of the perpendicular $\perp$ and parallel $\|$ components of polarisation. $\theta_{i}$ and $\theta_{i}^{\prime}$ are the angles of incidence on the AB face of the prism while the corresponding angles of refraction are, $\theta_{r}$ and $\theta_{i}^{\prime}, \theta_{s}$ and $\theta_{s}^{\prime}$ on the $\mathrm{AC}$ and $\mathrm{BC}$ faces of the prism.

The incident light intensity and loss of light due to scattering and absorption by the sample are given by $I_{0}$ and $I_{1}$. The polarisation angle, $\phi$ gives the difference between the transmission directions of the two polarisers.

\subsection{Least Squares Estimation}

The transmitted radiance sinusoid in Equation (5) has amplitude $\sqrt{A_{1}^{2}+B_{1}^{2}}=\frac{I_{t_{\max }}-I_{t_{\min }}}{2}$, and offset (DC level) $T_{1}=\frac{I_{t_{\max }}+I_{t_{\min }}}{2}$. The least squares fit for a sine function of known frequency [12] is used in order to compute $T_{1},\left(A_{1}^{2}+B_{1}^{2}\right)$ and $\beta$ from the values of $I_{t}(\phi)$ acquired experimentally and $\alpha_{e}, \triangle$. As has been mentioned the values for the transmitted light intensity are taken through the change in the polariser angle of the polaroid placed in front of the camera. It is evident from the above equations that the values of $\alpha_{e}$ and $\triangle$ are dependent on the ratios between $A_{1}, T_{1}$ and $A_{1}, B_{1}$, which show that the values for the complex refractive index are independent of the loss of light, due to the sample and the absolute intensity of the incident light.

\section{Experiments}

As mentioned earlier, the experiments were carried out in a dark room. The camera and the light sources are positioned at an angle to the object in the geodesic light dome using the experimental setup given in Figure (1). The samples are roughly divided into two categories 1 . samples with unknown refractive indices comprising of naturally occurring surfaces and painted surfaces and 2. samples of known refractive indices comprising of chemical suspensions. For naturally occurring surfaces, the experiments were carried out on a wrinkled and fresh apple, orange, pepper and potato while, for industrially produced surfaces the experiments were carried out on painted pieces of paper. The same paper was painted with the same paints forming various compositions by mixing with other materials such as: 1 . mineral blush, 2. talcum powder, 3 . cumin powder and 4. powdered coal, the sample of plain paint was later coated with a layer of vaseline. The orange has been chosen in order to test the method for the effect of the presence of natural indentations in the surface.As shown in the Figure (1) the material whose refractive index is to be determined is placed/applied at the base of the prism. As for the chemical compounds, suspension were created by mixing $1 \mathrm{~g}$ to $4 \mathrm{~g}$ of the compound in vaseline and IPA (isopropanol) with increments of $1 \mathrm{~g}$. The chemical suspensions were applied directly to the base of the prism so that the estimates are only of the material under study while for the experiments in the category of unknown refractive index, the samples were mounted on a cardboard mount. The aim here is to determine the effectiveness of the method for material variation and also a sample was included to test its effectiveness for non-planar surface. 


\subsection{Results for Estimating the Complex Refractive Index Using Ellipsometry}

The values for the real part of the complex refractive indices fall in the range $0<n<3$, where the non-physical outliers have been filtered. This corresponds to the parts of the surface in the case of rotting and wrinkled tissue that are not in contact with the surface of the prism. Errors arise due to the inter-reflection that occur because of the wrinkled surface resulting in unrealistic values and also to the non-physical values of the refractive index, which are less than unity. The potential sources of error are those that occur mainly due to the image acquisition process which result from camera jitter and human error in the positioning of the polariser filter, which also result in errors in the computation of the degree of polarisation. Additional sources of error in this experiment are due to the lack of contact between the material and the base of the prism also, in the case of the chemical suspensions created in IPA (which is volatile hence evaporates rapidly) when the IPA had significantly evaporated the coating started to crack and in case of the $4 \mathrm{~g}$ a gap was created between the suspension and the prism surface leading to an error that is caused by non-contact.

The suspensions were used in order to verify how the method performs for materials of known refractive indices. Some experiment were also conducted in order to determine whether increasing concentrations of the compounds have an effect on the computations of the complex refractive index especially the extinction coefficient. The results for the estimations of the complex refractive index for chemical suspension are given in Table (2). The concentrations of the solutes were changed in order to study the variation of the complex refractive index especially the extinction co-efficient. It was found out by repeated experimentation that the change in the imaginary part of the composite quantity of the complex refractive index was not large hence the values were almost constant. Referring to results in existing literature such changes can be experienced when the sample has aged [13] while in this case the images were taken within the span of a few minutes or a day at the maximum. The variation in the values can be attributed to the noise in the image acquisition process.

The results for the estimations of the complex refractive index for mosaics are given in Table (1). The mosaics were created by combining two different materials as, 1. Zinc Oxide and Cobaltus Sulphate by using vaseline as a solvent, 2. Dormant yeast in cold water and activating the yeast by making a suspension with sugar and warm water, and 3. Cobaltus Sulphate by using IPA and vaseline. The mosaics were created and experimented on such that the method could be tested to verify its ability to compute the complex refractive index effectively for two different materials in the same image.

For the mosaics of 1. Zinc Oxide and Cobaltus Sulphate and 2. Cobaltus Sulphate, the histograms for the real and imaginary parts of the refractive index are give in Figure (2) and (3). It is evident from the bi-modal histogram for the imaginary values that it signifies the two materials in the mosaic. Also, for the same material, the histogram is bi-modal as the suspensions were created in a solvent whose characteristics are different from those of the chemical hence, the two peaks (as the absorption differs for the materials). The histograms for the real values of the same material have a single high peak which falls around the value of the refractive index of the material. The chemical suspensions that were created did not have uniform dimensions especially, near the edges of the prism where the material thinned and did not cover the base completely and 
Table 1. The estimated Complex Refractive Indices for Mosaics using Ellipsometry

\begin{tabular}{|c|c|}
\hline Sample Material & Estimated Complex Refractive index 'n' \\
\hline Zinc Oxide and Cobaltus Sulphate & $1.54-0.743 \mathrm{i}$ \\
Active and Dormant Yeast & $1.90-1.481 \mathrm{i}$ \\
Cobaltus Sulphate & $1.70-0.964 \mathrm{i}$ \\
\hline
\end{tabular}

Table 2. The estimated Complex Refractive Indices for Chemical Suspensions using Ellipsometry

\begin{tabular}{|c|c|c|c|}
\hline Sample Material & Quantity $(\mathrm{g})$ & Solvent & Estimated Complex Refractive index 'n' \\
\hline Zinc Oxide & 1 & IPA & $1.62-0.318 \mathrm{i}$ \\
Zinc Oxide & 2 & IPA & $1.47-0.907 \mathrm{i}$ \\
Zinc Oxide & 3 & IPA & $1.53-0.003 \mathrm{i}$ \\
Zinc Oxide & 4 & IPA & $1.69-0.617 \mathrm{i}$ \\
Zinc Oxide & 1 & Vaseline & $1.54-1.432 \mathrm{i}$ \\
Zinc Oxide & 2 & Vaseline & $1.55-0.727 \mathrm{i}$ \\
Zinc Oxide & 3 & Vaseline & $1.60-0.787 \mathrm{i}$ \\
Zinc Oxide & 4 & Vaseline & $1.56-0.199 \mathrm{i}$ \\
Cobaltus Sulphate & 1 & IPA & $1.58+0.858 \mathrm{i}$ \\
Cobaltus Sulphate & 2 & IPA & $1.48-0.347 \mathrm{i}$ \\
Cobaltus Sulphate & 3 & IPA & $1.48-0.372 \mathrm{i}$ \\
Cobaltus Sulphate & 4 & IPA & $1.80-0.216 \mathrm{i}$ \\
Cobaltus Sulphate & 1 & Vaseline & $2.01-0.572 \mathrm{i}$ \\
Cobaltus Sulphate & 2 & Vaseline & $1.93-0.496 \mathrm{i}$ \\
Cobaltus Sulphate & 3 & Vaseline & $1.95-0.359 \mathrm{i}$ \\
Cobaltus Sulphate & 4 & Vaseline & $1.94-0.364 \mathrm{i}$ \\
\hline
\end{tabular}

Table 3. The estimated Complex Refractive indices using Ellipsometry

\begin{tabular}{|c|c|}
\hline Sample Material & Estimated Complex Refractive index 'n' \\
\hline Colour Patch & $1.62+1.207 \mathrm{i}$ \\
Colour Patch Coated with Vaseline & $1.42+1.308 \mathrm{i}$ \\
Cumin Powder & $1.70+1.213 \mathrm{i}$ \\
Powdered Coal & $1.79+1.176 \mathrm{i}$ \\
Talcum Powder & $1.37+1.342 \mathrm{i}$ \\
Mineral Foundation & $1.77+1.118 \mathrm{i}$ \\
Fresh Apple & $1.66+1.162 \mathrm{i}$ \\
Wrinkled Apple & $1.62+1.335 \mathrm{i}$ \\
Potato & $1.40+1.952 \mathrm{i}$ \\
Orange & $1.66+1.363 \mathrm{i}$ \\
Pepper & $1.78+1.929 \mathrm{i}$ \\
\hline
\end{tabular}

with non-uniform thickness. For the histograms of real values of the refractive index, for the mosaic of Zinc Oxide and Cobaltus Sulphate two to three peaks can be observed. They can be attributed to the two materials comprising the mosaic, but it is not a very clear determinant of the refractive index. The average estimated values for the complex refractive index are in compliance with the existing published values of the materials. 


\subsection{Limitations}

The limitations of the methods proposed thus far are: low accuracy due to the use of large amount of tissue and complicated setup or specific depth measurements of the tissue. In our experiments the method is simple and relatively easy to set up as the equipment being used is not specialised for the estimation of complex refractive index (the sample's images have been taken at intervals of $10^{\circ}$ and in some cases at $5^{\circ}$ ), neither is the tissue thickness consistent but, has varied with the different samples. The computed refractive indices of the experimental samples are given in the Table (3). It can be seen from the table that the computed value for the refractive index of vaseline coated paint, has an error of $4.15 \%$, while the values for the refractive index of apple are quite similar but, there is a difference in the computation of the extinction co-efficient hence more light is being absorbed by the wrinkled apple where the tissue water content is low as compared to the fresh apple.

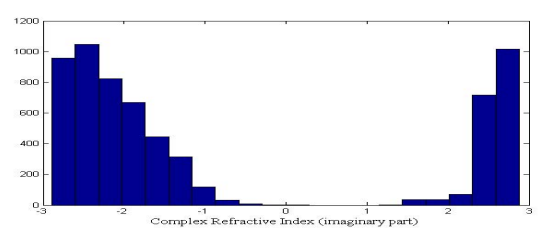

(a)

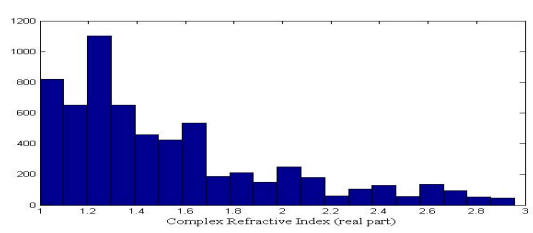

(b)

Fig. 2. The histogram of the estimation for the real and imaginary parts of the complex refractive index of the material mosaics using Ellipsometry of a. Zinc Oxide and Cobaltus Sulphate.

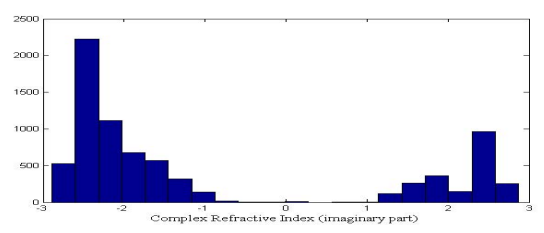

(a)

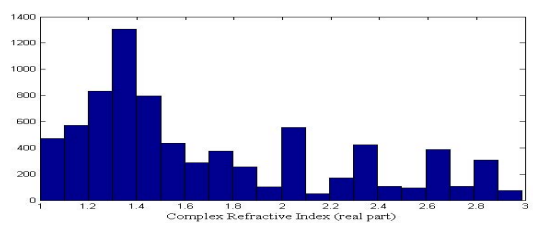

(b)

Fig. 3. The histogram of the estimation for the real and imaginary parts of the complex refractive index of the material mosaics using Ellipsometry of Cobaltus Sulphate in IPA and Vaseline

\section{Conclusion}

In this paper we show how complex refractive index can be computed by making use of ellipsometry. Rather than using a goniometer to perform the required calibrated photometric measurements, we use a geodesic light dome, using a digital camera together with polarisation measurements. Using this more simple experimental setup complex refractive index can be computed using dimensionless quantities that do not require photometric calibration. When used on mosaics of different materials, the method can be used to detect changes in material composition. 
The method needs to be refined in various way. For instance in the data acquisition process, there is a problem associated with shadowing from the prism faces. The solution could be the use of a prism with a large base area so that the image can be captured without the shadowing effect. Another drawback of our method is that it requires a planar surface sample that has to be in complete contact with the base of the prism. The case of loss-of-contact leads to invalid complex refractive index values. However, the method that has been used in this paper does not require large tissue samples. The proposed method can be used to segment surfaces into regions of different composition or to inspect for surface lesions or flaws.

\section{References}

1. Bolin, F.P., Preuss, L.E., Taylor, R.C., Ference, R.J.: Refractive Index Estimation Using Polarisation and Photometric Stereo. Applied Optics, Optical Society of America (1989)

2. Tsenova, V., Stoykova, E.V.: Refractive index measurement in human tissue samples. In: Proceedings of SPIE (2003)

3. Dirckx, J.J.J., Kuypers, L.C., Decraemer, W.F.: Refractive index of tissue measured with confocal microscopy. Journal of Biomedical Optics (2005)

4. Greenleaf, J.F., Johnson, S.A., Lent, A.H.: Measurement of spatial distribution of refractive index in tissue by ultrasonic computer assisted tomography. Ultrasound in Medicine and Biology (1978)

5. Hunter, W.R.: Error in using the reflectance vs angle of incidence method for measuring optical constants. Journal of the Optical Society of America (1965)

6. Ding, H., Lu, J.Q., Wooden, W.A., Kragel, P.J., Hu, X.: Refractive indices of human skin tissues at eight wavelengths and estimated dispersion relations between 300 and $1600 \mathrm{~nm}$. Journal Physics in Medicine and Biology (2006)

7. Shashar, N., Cronin, T.W., Wolff, L.B., Condon, M.A.: The polarisation of light in a tropical rain forest. The association for Tropical Biology and Conservation (1998)

8. Land, M.F.: Old twist in a new tale. Nature (1993)

9. Cooper, P., Thomas, M.: Geodesic Light Dome. Department of Computer Science. University of York, UK (2010), http://www-users.cs.york.ac.uk/-pcc/Circuits/dome

10. Lai, J.C., Zhang, Y.Y., Li, Z.H., Jiang, H.J., He, A.Z.: Complex refractive index measurement of biological tissues by attenuated total reflection ellipsometry. Applied Optics (2010)

11. Saman, G., Hancock, E.R.: Robust Computation of the Polarisation Image. In: International Conference on Pattern Recognition (2010)

12. IEEE: IEEE Standard for Digitizing Waveform Recorders, IEEE Std 1057-1994 (1994)

13. Sato, K., Manabe, T., Polivka, J., Ihara, T., Kasashima, Y., Yamaki, K.: Measurement of the complex refractive index of concrete at $57.5 \mathrm{GHz}$. IEEE Transactions on Antennas and Propagation (1996) 